\title{
Role of TRPM7 in cardiac fibrosis: A potential therapeutic target (Review)
}

\author{
FENG HU $^{1 *}$, MEIYONG LI ${ }^{2 *}$, FENGYU HAN ${ }^{1}$, QING ZHANG ${ }^{3}$, \\ YUHAO ZENG $^{4}$, WEIFANG ZHANG ${ }^{*}$ and XIAOSHU CHENG ${ }^{1,6^{*}}$
}

\author{
Departments of ${ }^{1}$ Cardiovascular Medicine and ${ }^{2}$ Laboratory Medicine, The Second Affiliated Hospital of Nanchang University, \\ Nanchang, Jiangxi 330006; ${ }^{3}$ Department of Cardiology, The Union Hospital of Tongji Medical College, \\ Huazhong University of Science and Technology, Wuhan, Hubei 430022; ${ }^{4}$ Department of Medical Education, \\ The Second Clinical Medical College of Nanchang University; ${ }^{5}$ Department of Pharmacy and \\ ${ }^{6}$ Center for Prevention and Treatment of Cardiovascular Diseases, The Second Affiliated Hospital of \\ Nanchang University, Nanchang, Jiangxi 330006, P.R. China
}

Received April 4, 2020; Accepted December 4, 2020

DOI: $10.3892 / \mathrm{etm} .2020 .9604$

\begin{abstract}
Cardiac fibrosis is a hallmark of cardiac remodeling associated with nearly all forms of heart disease. Clinically, no effective therapeutic drugs aim to inhibit cardiac fibrosis, owing to the complex etiological heterogeneity and pathogenesis of this disease. A two-in-one protein structure, a ubiquitous expression profile and unique biophysical characteristics enable the involvement of transient receptor potential melastatin-subfamily member 7 (TRPM7) in the pathogenesis and development of fibrosis-related cardiac diseases, such as heart failure (HF), cardiomyopathies, arrhythmia and hyperaldosteronism. In response to a variety of stimuli, multiple bioactive molecules can activate TRPM7 and related signaling pathways, leading to fibroblast proliferation, differentiation and extracellular matrix production in cardiac fibroblasts. TRPM7-mediated $\mathrm{Ca}^{2+}$ signaling and TGF- $\beta 1$ signaling pathways are critical for the formation of fibrosis. Accumulating evidence has demonstrated that TRPM7 is a potential pharmacological target for halting the development of fibrotic cardiac diseases. Reliable drug-like molecules for further development of high-affinity in vivo drugs targeting TRPM7 are urgently needed. The present review discusses the widespread and significant role of TRPM7 in cardiac fibrosis and focuses on
\end{abstract}

Correspondence to: Dr Weifang Zhang, Department of Pharmacy, The Second Affiliated Hospital of Nanchang University, 1 Minde Road, Nanchang, Jiangxi 330006, P.R. China

E-mail: z_weifang@163.com

Professor Xiaoshu Cheng, Department of Cardiovascular Medicine, The Second Affiliated Hospital of Nanchang University, 1 Minde Road, Nanchang, Jiangxi 330006, P.R. China

E-mail: xiaoshumenfan@126.com

Key words: cardiac fibrosis, cardiac remodeling, transient receptor potential melastatin-subfamily member 7 its potential as a therapeutic target for alleviating heart fibrogenesis.

\section{Contents}

1. Introduction

2. Interacting proteins

3. Physiological roles of transient receptor potential melastatin-subfamily member 7 (TRPM7) in the heart

4. Pathological functions of TRPM7 in cardiac fibrosis

5. Modulators for TRPM7

6. Implications and conclusion

\section{Introduction}

Cardiac fibrosis is a hallmark of cardiac remodeling associated with nearly all forms of heart disease, such as heart failure (HF), myocardial infarction (1), dilated and ischemic cardiomyopathies (ICMs) (2), arrhythmia (3) and hyperaldosteronism (4). The adult mammalian heart has negligible regenerative capacity, and cardiac repair is dependent on the clearance of dead cells and the formation of scar tissue to help preserve myocardial structural and functional integrity (5). Meanwhile, unrestrained tissue repair can result in pathological fibrosis, causing detrimental abnormalities $(5,6)$. Pathological cardiac fibrosis is orchestrated predominantly by myofibroblasts, which are activated fibroblasts characterized by overproduction of growth factors, cytokines, chemokines, proteases and extracellular matrix (ECM) proteins. Moreover, myofibroblasts are particularly responsive to proinflammatory cytokines, including TNF- $\alpha$, IL-1, IL- 6 and TGF- $\beta$; vasoactive peptide angiotensin II (Ang II), endothelin-1, atrial natriuretic peptide and brain natriuretic peptide (5-7). The sources of these activated fibroblasts that accumulate in response to various pathological insults, such as myocardial injury, oxidative stress, mechanical stretch, autocrine-paracrine mediators 
and inflammatory stimuli, remain under active investigation (8). Lineage tracing has recently been utilized in cardiac fibrosis studies, and numerous cells, including resident fibroblasts (9), endothelial cells (ECs) (10) or epicardial cells (11), hematopoietic bone marrow-derived macrophages (12) and perivascular cells (13), have been proposed as precursors of the fibroblast population in the injured heart. Thus, the differentiation of quiescent fibroblasts into active matrix-producing myofibroblasts is a key step in disease progression (5). Cardiac fibrosis induces adverse structural remodeling of the myocardium, resulting in abnormalities in cardiac conduction, loss of contractility, and hardening of the ventricular walls (5). Thus, understanding the underlying mechanisms of cardiac fibrosis will facilitate the development of innovative treatment strategies to hinder pathological fibrosis.

The TRPM subfamily plays a crucial role in various physiological and pathological conditions such as those related to sensory or renal physiology, cancer, cardiac health and neuronal development (14). The subfamily contains eight isoforms that form four subfamilies of TRPM channels: TRPM1/3, TRPM2/8, TRPM4/5, and TRPM6/7. Among these, TRPM7, TRPM2 and TRPM6 are unique because they are bifunctional heteromeric ion channels containing functional enzymatic domains in their highly varied C-terminal segments (15). The TRPM2 channel has an enzymatic domain similar to Nudix hydrolase, cleaving monodinucleotidic polyphosphates (15). On the other hand, the TRPM6 and TRPM7 channels contain kinase domains and are classified as atypical alpha protein kinases (15). The double activity as channels and enzymes of these two proteins classifies them either as chanzymes or channel-kinases (16) (Fig. 1). TRPM7 and its close homologue TRPM6 are present in the tetrameric form, with each subunit consisting of six transmembrane segments, and are both permeable to calcium $\left(\mathrm{Ca}^{2+}\right)$, magnesium $\left(\mathrm{Mg}^{2+}\right)$ and zinc cations $\left(\mathrm{Zn}^{2+}\right)(17)$. The disruption of TRPM6 kinase phosphorylation activity reintroduces $\mathrm{MgATP}$ sensitivity to the heteromeric channel, which is similar to that of TRPM7, supporting the notion that TRPM6 kinase plays a critical role in the control of the TRPM7/6 channel complex (18).

The TRPM subfamily has been shown to have highly differing modes of activation, cation selectivity and tissue distribution (19) and play crucial roles in various physiological and pathological conditions such as those related sensory or renal physiology, cancer, cardiac health and neuronal development (14). TRPM6 is mainly expressed in intestinal and renal epithelia, and TRPM2 expression is highest in the brain and bone marrow (20). In contrast to TRPM6 and TRPM2, which have specific expression patterns, TRPM7 is widely distributed in the central nervous system as well as in the periphery, with the highest expression levels in the heart, pituitary, bone and adipose tissue (20). TRPM7 expression in cardiac fibroblasts (CFs) has been verified by immunocytochemistry (21). Two of the characteristics of TRPM7 channels, specifically, their non-voltage-gated design and $\mathrm{Ca}^{2+}$ permeability, suggest that they may have significant pathological and physiological functions in various cells, particularly in non-excitable cells such as CFs (21).

Their two-in-one protein structure, ubiquitous expression profile and unique biophysical characteristics that enable divalent ion transport results in the involvement of
TRPM7 involvement in a number of pathophysiological processes (22-34), including $\mathrm{Mg}^{2+}(25,26)$ and $\mathrm{Ca}^{2+}(27)$ homeostasis, immune system homeostasis (28), embryonic development $(25,29)$, hyperaldosteronism (4), cardiovascular inflammation and fibrosis (22), hypertension (23), diabetes $(30,31)$, cerebral ischemia and hypoxia (32), airway remodeling (33) and tumorigenic activity $(34,35)$. A lack of either TRPM6 or TRPM7 was found to be embryonically lethal, and several Mg-related diseases have been linked to mutations in these channels $(22,23,29,36,37)$. Inhibition of TRPM2has been indicated to protect against renal fibrosis and inflammation (38), whereas chanzyme TRPM7 has been demonstrated to protect against cardiovascular fibrosis and inflammation (22). Since TRPM7 expression is highest in the heart, previous studies have demonstrated that TRPM7 plays a crucial role in cardiac fibrosis-related diseases $(21,22)$. The present review will focus on recent developments in understanding the role of TRPM7 in cardiac fibrogenesis and the potential of TRPM7 as a therapeutic target for antifibrotic drug development.

\section{Interacting proteins}

TRPM7 represents a constitutively active ion channel that is heavily regulated by a variety of physiological feedback mechanisms. One of the most important regulatory factors of channel activity is free intracellular $\mathrm{Mg}^{2+}$ (39), which opens channels and thus result in ion movements. Detailed biophysical examination revealed that native TRPM7 in excised patches has two conductance states at 39 picosiemens (pS) and $186 \mathrm{pS}$, with both reversibly inhibited by $\mathrm{Mg}^{2+}(40)$.

Information on proteins interacting with TRPM7 remain scarce, even for the kinase domain. A study has shown that receptor-stimulated activation of phospholipase (PLC causes inhibition of TRPM7 channel activity through localized phosphatidylinositol 4,5-bisphosphate $\left(\mathrm{PIP}_{2}\right)$ hydrolysis (41). Furthermore, hypomagnesemic conditions increased TRPM7-kinase-regulated Ser/Thr phosphorylation in the $\mathrm{C} 2$ domain of PLC $\gamma 2$, leading to reduced $\mathrm{Ca}^{2+}$ signaling (42), whereas the $\alpha$-kinase domain activates downstream target proteins involved in cytoskeleton organization, cell proliferation, inflammatory responses and vascular contraction, among other properties $(39,43)$. The involvement of TRPM7 kinase in cell motility and adhesion has been linked to its ability to phosphorylate the assembly domains of non-muscle myosin IIA, IIB, and IIC and ATP-dependent motor proteins involved in actomyosin-based cell motility (44-46). Annexin $\mathrm{A} 1, \mathrm{Ca}^{2+}$-dependent membrane-binding protein with the ability to promote membrane fusion, is also phosphorylated by the TRPM7 kinase, providing a possible link to TRPM7's known involvement in cell growth and apoptosis $(47,48)$.

TRPM7 has autophosphorylation residues, and cleavage of the $\alpha$-kinase results in the release of fragments that bind to transcription factors, leading to epigenetic modifications (49). Phosphomapping by mass spectrometry identified 47 autophosphorylation sites on TRPM7, the majority of which are located in the Ser/Thr-rich domain N-terminal of the kinase region (16). This part of the TRPM7 region is thought to control kinase substrate binding (16). The TRPM7 kinase specifically phosphorylates Ser and Thr residues 

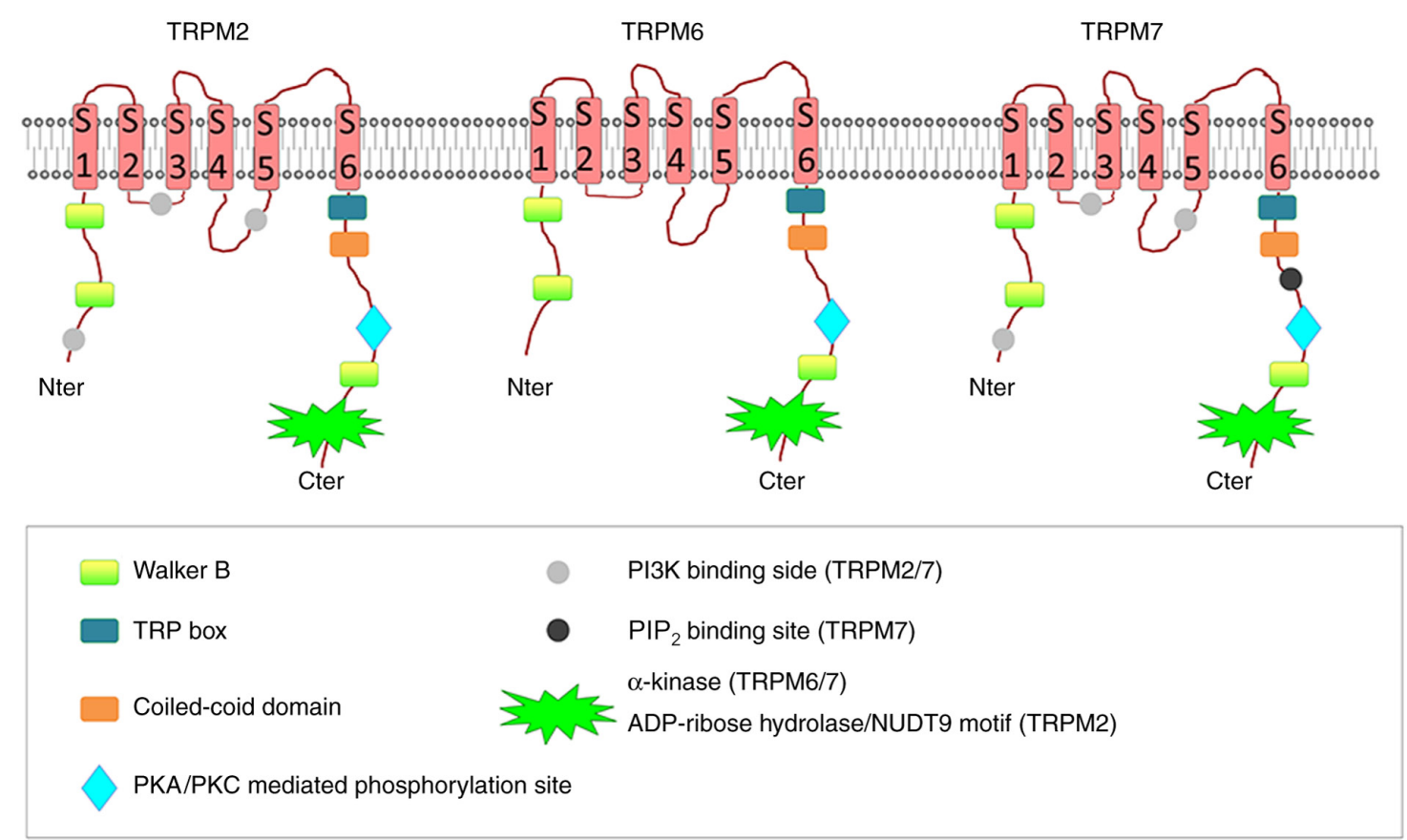

Figure 1. Schematic structure of TRPM2, TRPM6 and TRPM7 proteins. Nter, N-terminal; Cter, C-terminal; TRPM, transient receptor potential melastatin; $\mathrm{PIP}_{2}$, phosphatidylinositol 4,5-bisphosphat; PKA, protein kinase A; PKC, protein kinase C; NUDT9, ADP-ribose pyrophosphatase.

in a $\mathrm{Mg}^{2+}$-dependent manner (50). It autophosphorylates itself and phosphorylates myelin basic protein as well as histone H3 (51). At least two of the identified autophosphorylation sites (S1511 and S1567) do not seem to influence channel behavior (51). These proteolytically TRPM7-cleaved kinase fragments (M7CKs) translocate to the nucleus and bind multiple components of chromatin remodeling complexes, leading to epigenetic modifications and thereby influencing gene expression profiles (49). Furthermore, free cytosolic $\mathrm{Zn}^{2+}$ is TRPM7-dependent and regulates M7CK binding to transcription factors containing zinc-finger domains (49). These findings suggested that TRPM7-mediated modulation of intracellular $\mathrm{Zn}^{2+}$ concentration couples ion channel signaling to epigenetic chromatin covalent modifications that affect gene expression patterns (49). Deletion of TRPM7 in T-cells reduces apoptosis in response to Fas stimulation, while caspase-dependent cleavage at D1510 leads to separation of TRPM7's kinase domain from the channel without affecting the functionality of the kinase but enhancing ion channel activity (52).

The channel and/or enzyme domain of TRPM7 is relevant to cardiac fibrosis. TRPM7 is involved in Ang II-induced cardiac fibrosis development by mediating $\mathrm{Mg}^{2+}$ and $\mathrm{Ca}^{2+}$ influx (53). TRPM $7^{+/ \Delta^{k i n a s e}}$ mice exhibit cardiovascular inflammation and fibrosis associated with $\left[\mathrm{Mg}^{2+}\right]_{\mathrm{i}}$ deficiency, abnormal macrophage activation, inflammatory cell infiltration, and increased signaling through Smad3, calpain-II and Stat1 (22). The kinase domain may influence the channel domain supported by the tissue, and cellular $\mathrm{Mg}^{2+}$ levels were lower, while TRPM7 channel phosphorylation was reduced in TRPM $7^{+/ \Delta^{k i n a s e}}$ mice compared with control counterparts (22). Other studies have also suggested that the coexistence of the TRPM7 channel and kinase is functionally relevant; for example, channel-mediated $\mathrm{Mg}^{2+}$ influx is required for TRPM7 kinase activity, and thus regulates RhoA activity and subsequent transcriptional activation in hepatocellular carcinoma cells (34). The TRPM7 kinase inhibitor TG100-115 suppressed ion channel activity in breast cancer cells (54).

\section{Physiological roles of TRPM7 in the heart}

TRPM7 is highly expressed in the adult heart and is first expressed in the embryonic myocardium (25). The physiological consequences of cardiac-targeted TRPM7 deletion depend on the timing of TRPM7 disruption during cardiogenesis (29). Early cardiac TRPM7 deletion before embryonic day 9 causes HF and death by embryonic day 11.5 due to myocardial fragility (29). Remarkably, mice with TRPM7 deletion late in cardiogenesis, at approximately embryonic day 13 , exhibit normal adult ventricular structures and functions (29). Genetic deletion of TRPM7 at an intermediate developmental timepoint during mouse cardiogenesis alters the myocardial transcriptional profile and variably damages adult ventricular function, inducing atrioventricular block, impaired repolarization and ventricular arrhythmias (29). Interestingly, cardiac-targeted TRPM7 deletion during the intermediate and late stages (approximately embryonic days 11.5-13) results in significant interstitial ECM deposition and interstitial fibrosis in hearts at 6-8 months (29). Consistent with the cardiomyopathic phenotype, researchers also observed expected enrichment in genes upregulated in the ECM, ECM receptor interactions and pressure-overload murine heart disease (29). These results indicated that subtle differences in the timing of TRPM7 disruption led to significantly different cardiac phenotypes. TRPM7 is dispensable in the adult ventricular myocardium under basal conditions but is critical for myocardial proliferation during early cardiogenesis and fibrosis during intermediate-and-late cardiogenesis. 
In addition, TRPM7 in other organs can also affect heart function, especially in inflammation and fibrosis $(4,22)$. TRPM7-dificient mice with deletion of the kinase domain (TRPM7 $7^{+/ \Delta^{\mathrm{kinase}}}$ ) showed significant cardiac hypertrophy, fibrosis and inflammation (22). Furthermore, TRPM $7^{+/ \Delta^{\mathrm{kinase}}}$ mice exhibit distinct pro-inflammatory and pro-fibrotic cardiovascular and renal phenotype linked to macrophage activation, increased signaling through Smad3, calpain-II and Stat1 and cellular hypomagnesaemia (22). The different physiological consequences between cardiac-targeted TRPM7 deletion and systemic deletion of the kinase domain alone may be due to macrophages from TRPM $7^{+/ \Delta^{k i n a s e}}$ mice producing soluble factors that promote a pro-fibrotic phenotype in CFs through $\mathrm{Mg}^{2+}$-dependent mechanisms (22).

Other studies suggested that TRPM7 activation causes dysregulated immune responses and inflammation, and that TRPM7 inhibition may have therapeutic potential in pro-inflammatory diseases and immune hypersensitivity $(55,56)$. These discrepancies likely depend on the relative contributions of the TRPM7 channel vs. the kinase domain and highlight the complexity of the system. These results provided diagnostic insight into putative mutations in the TRPM7 gene in patients with unexplained structural or electrophysiological heart disease.

\section{Pathological functions of TRPM7 in cardiac fibrosis}

Pathological cardiac fibrosis in response to myocardial injury and/or chronic alterations of myocardial loading conditions increase myocardial wall stiffness and disrupt the order of myocardial structure, which is a requisite for normal cardiac output and electrical conduction, culminating in chamber dilatation, cardiomyocyte hypertrophy and apoptosis and ultimately leading to the development of $\mathrm{HF}$ and increased arrhythmogenicity (57). Atrial fibrosis is also a common pathological change in elderly people that is strongly associated with the perpetuation of atrial fibrillation (AF) (58). The biochemical mechanisms of cardiac fibrosis involve impaired $\mathrm{Ca}^{2+}$ or $\mathrm{Mg}^{2+}$ homeostasis, oxidative stress, hemodynamic abnormalities and activation of neurohormones, including Ang II, TGF- $\beta 1$ and endothelin-1 $(2,5,6,22,58,59)$. The production of increased quantities of ECM proteins by CFs leads to tissue fibrosis, which can impair both mechanical and electrical function of the heart, contributing to AF and HF (60). Previous studies have shown that TRPM7 is the only calcium channel expressed on the cell membrane of CFs (61), and that $\mathrm{Ca}^{2+}$ signaling is closely associated with the initiation of fibrosis (62-64). These findings suggested that TRPM7 may be the one of the most potent fibrosis factors, playing an important role in the molecular mechanism and pathological processes in cardiac fibrosis (Fig. 2).

TRPM7-mediated $\mathrm{Ca}^{2+}$ signaling during cardiac fibrosis. $\mathrm{Ca}^{2+}$, which is influenced by TRPM7, is critically involved in controlling cell function including cell proliferation, growth, secretion, migration, differentiation and death (65). $\mathrm{Ca}^{2+}$ influx is necessary for the biological functions of CFs during collagen synthesis (66). The expression and current of TRPM7, but not of TRPM4, are increased in human atrial specimens from patients with AF compared with healthy controls (61). TRPM7-mediated $\mathrm{Ca}^{2+}$ influx is also significantly increased in atrial fibroblasts from patients with AF (61). TRPM7 is the molecular basis of the main $\mathrm{Ca}^{2+}$ permeable channel in human AF, which is the hallmark of atrial structural remodeling (67). Atrial structural remodeling is one of the most important fundamental mechanisms underlying the perpetuation of $\mathrm{AF}$ and contributes synergistically with electrical and neural remodeling to the AF substrate $(3,58)$. Previous studies have shown that TRPM7 is the only calcium channel expressed on the cell membrane of CFs (61). Knocking down TRPM7 largely eliminates endogenous TRPM7 currents and $\mathrm{Ca}^{2+}$ influx in AF (67).

Mechanistically, TRPM7-mediated $\mathrm{Ca}^{2+}$ influx mediates atrial fibroblasts differentiation into myofibroblasts, thus promoting atrial fibrosis (67). Myofibroblasts present with the characteristics of both smooth muscle cells and fibroblasts (66). In normal hearts, myofibroblasts are found only in valves (66). During tissue damage repair, myofibroblasts participate in two stages: Fiber formation and ECM remodeling in the fibrosis cascade reaction (66). TGF- $\beta 1$ can induce the transformation of atrial fibroblasts into myofibroblasts, simultaneously upregulating TRPM7 (67). Inhibition of TRPM7-mediated $\mathrm{Ca}^{2+}$ influx renders fibroblasts less sensitive to TGF- $\beta 1$-induced proliferation and differentiation, indicating that TRPM7-mediated $\mathrm{Ca}^{2+}$ signal is necessary for TGF- $\beta 1$ elicited fibrogenesis (67). Inhibition of TRPM7 may prove to be an effective approach to reduce fibroblast differentiation and therefore attenuate fibrosis during AF.

Similar to the aforementioned roles in AF, TRPM7-mediated $\mathrm{Ca}^{2+}$ signaling may also mediate ventricular fibrosis. Previous studies have shown that TRPM7 expression and currents were upregulated in isoproterenol (ISO)-induced cardiac fibrosis in vitro and in vivo (68). Recently, TRPM7 was demonstrated to be increased in left ventricular tissue samples isolated from the explanted hearts of end-stage patients with HF (69). HF is characterized by impaired $\mathrm{Ca}^{2+}$ homeostasis/contraction, markedly prolonged $\mathrm{Ca}^{2+}$ transients and impaired restoration of low diastolic $\mathrm{Ca}^{2+}$ concentrations (70). Increased internal flow of extracellular $\mathrm{Ca}^{2+}$ to the cell activates and initiates biological signaling cascades, leading to the secretion of a large quantity of pro-inflammatory and pro-proliferative cytokines and ECM proteins, ultimately giving rise to cardiac interstitial fibrosis (70). In adult rat ventricular CFs, 2-aminoethyl diphenylborinate (2-APB, an TRPM7 inhibitor) or TRPM7 knockdown by short hairpin RNA abolished $\mathrm{Ca}^{2+}$ influx induced by Ang II (53). Furthermore, 2-APB inhibited the increase of myocardial connective tissue growth factor, $\alpha$-smooth muscle actin (SMA) expression and CF proliferation induced by Ang II (53). To date, the mechanism of TRPM7-mediated $\mathrm{Ca}^{2+}$ signaling in cardiac fibrosis in $\mathrm{HF}$ has not been reported and requires further investigation.

TRPM7 is involved in hypomagnesemia during cardiac fibrosis. $\mathrm{Mg}^{2+}$ is the second-most abundant cation in mammalian cells and an essential cofactor in numerous enzymatic reactions. $\mathrm{Mg}^{2+}$ influences cell growth processes associated with remodeling and fibrosis, which are characteristic features of vascular damage in hypertension, atherosclerosis and diabetes (71). At the subcellular level, these effects occur at least partly via $\mathrm{Mg}^{2+}$-dependent regulation of mitogen-activated protein kinases, tyrosine kinases and reactive oxygen 

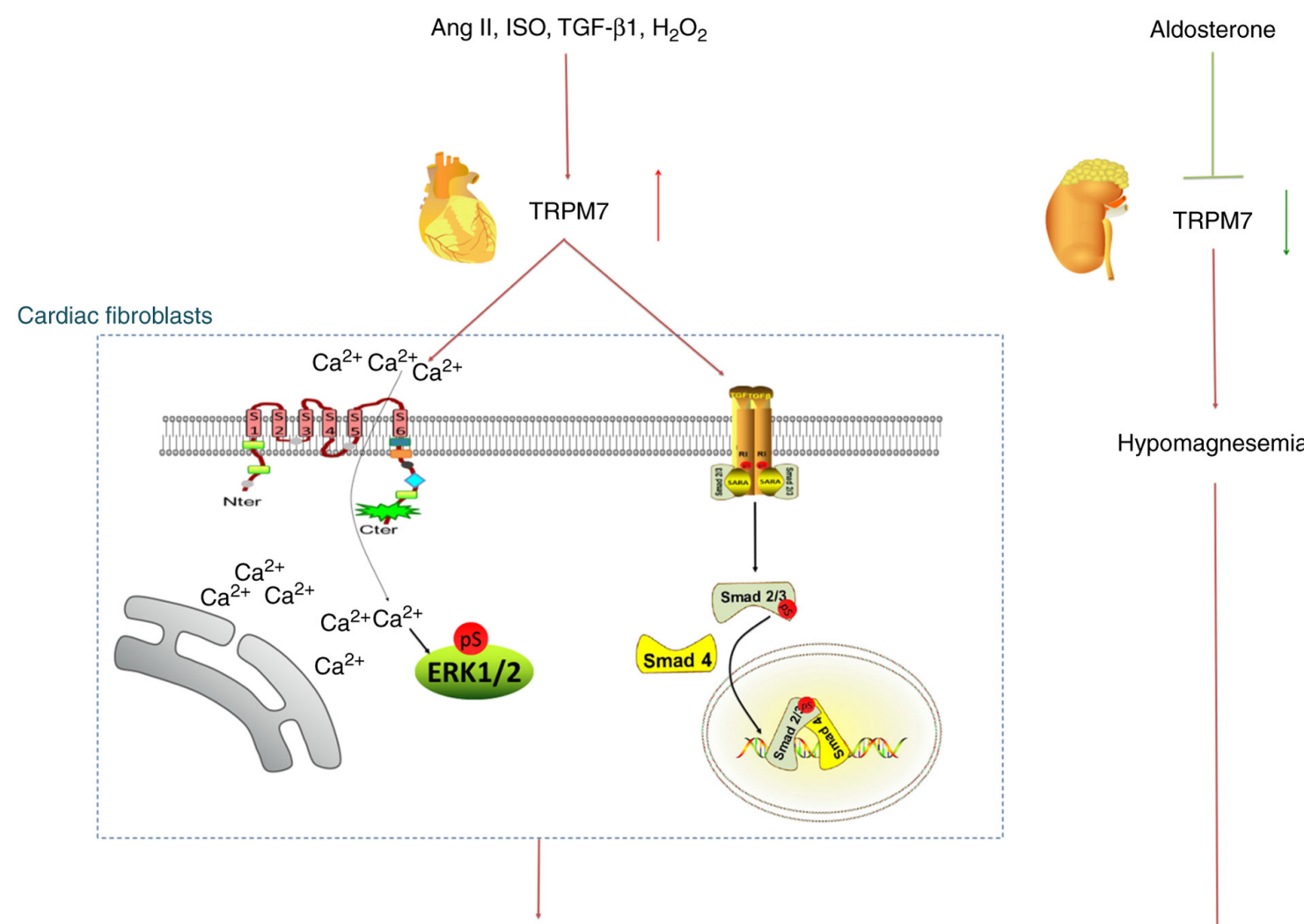

Proliferation and differentiation to myofibroblasts

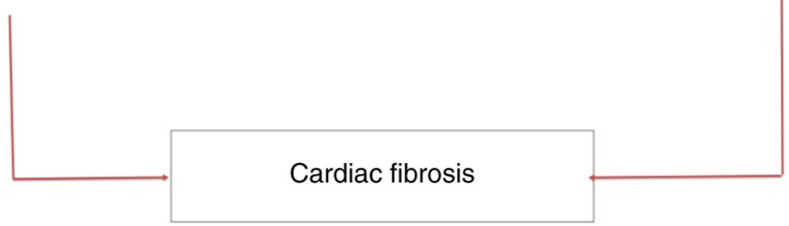

Hypomagnesemia

Figure 2. Schematic diagram illustrating the role of TRPM7 in the development and pathogenesis of cardiac fibrosis. TRPM, transient receptor potential melastatin; Ang II, angiotensin II; Nter, N-terminal; Cter, C-terminal; ISO, isoproterenol; pS, phosphorylation.

species, which are important signaling molecules involved in vascular smooth muscle cell proliferation, fibrosis and inflammation (72). Human microvascular endothelial cell growth inhibition by low magnesium is correlated with an increase in $\mathrm{P}_{21}$ or $\mathrm{P}_{27}$ kip, which are inhibitors of cyclin-dependent kinase (73).

TRPM7 is a key modulator of $\mathrm{Mg}^{2+}$ homeostasis, whose basal activity is regulated by intracellular levels of $\mathrm{Mg}^{2+}$ and MgATP (37). The role of TRPM7-mediated $\mathrm{Mg}^{2+}$ signaling in vascular homeostasis has been widely reported (74) but is less reported in the heart, especially in cardiac fibrosis. Recently, researchers utilized a particular DMEM $\left(\mathrm{Ca}^{2+}-\right.$ and $\mathrm{Mg}^{2+}$-free DMEM) supplemented with only calcium or only magnesium. The increase in $\alpha$-SMA and fibronectin expression induced by Ang II were abolished without calcium or magnesium, and the same outcome was observed for CF proliferation, indicating that both cations are required for the fibrosis response induced by Ang II, and that neither cation can be omitted (53). However, the association between TRPM7 and $\mathrm{Mg}^{2+}$ signaling in CFs requires further investigation.

In TRPM7 kinase-deficient mice, Ang II-induced cardiac hypertrophy, interstitial fibrosis and left ventricular dysfunction are amplified and associated with hypomagnesemia, inhibited TRPM7 kinase expression/signaling and pro-inflammatory vascular responses (23). Unlike the pro-cardiac fibrosis role of TRPM7 in mediating $\mathrm{Ca}^{2+}$ signaling, findings from animal models indicated that TRPM7 activation and increased $\mathrm{Mg}^{2+}$ influx protected against vascular and cardiac fibrosis (75). Hyperaldosteronism is associated with hypertension, cardiovascular fibrosis and electrolyte disturbances, including hypomagnesemia (76). Aldosterone modulates renal TRPM7 expression, which could be important in altered $\mathrm{Mg}^{2+}$ homeostasis associated with hyperaldosteronism (76). In a hyperaldosteronistic mouse model, aldosterone decreased the expression of renal TRPM7 without affecting TRPM6 and mediated blood pressure-independent renal and cardiovascular fibrosis and inflammation via $\mathrm{Mg}^{2+}$-sensitive pathways (4). $\mathrm{Mg}^{2+}$ supplementation normalizes TRPM7 mRNA expression and attenuates cardiac fibrosis (4). Therefore, $\mathrm{Mg}^{2+}$ supplementation may have an underlying therapeutic benefit in aldosterone-associated cardiac fibrosis.

TRPM7 is involved in oxidative stress during cardiac fibrosis. TRPM7 can be activated by oxidative stress-related pathologies, such as Alzheimer's disease, anoxia, ischemia/reperfusion injury and diabetes $(24,32,77)$. Reactive 
oxygen species-mediated oxidative stress is also well known to be the main contributor to cardiac injury and is involved in cardiac remodeling (78). In response to pathological stimuli such as oxidative stress and inflammation, CFs can differentiate into myofibroblasts to initiate myocardial fibrogenesis (79). In explanted human hearts with idiopathic dilated cardiomyopathy, ventricular tachycardia is associated with greater cardiomyocyte hypertrophy, myocardial fibrosis, oxidative stress and increased expression of TRPM7 (80). Previous studies have shown that TRPM7 is upregulated in rat models of myocardial ischemia and reperfusion (81). TRPM7 mRNA levels are downregulated in left atrial and left ventricular samples from patients with ICM, and this change has an inverse relationship with ventricular dysfunction (82). TRPM7 also contributed to $\mathrm{H}_{2} \mathrm{O}_{2}$-induced cardiac fibrosis by mediating $\mathrm{Ca}^{2+}$ influx and ERK1/2 activation in rat primary cardiac fibroblasts, and the blockade or silencing of TRPM7 inhibited myocardial fibrogenesis (83). Wu et al (68) found that microRNA (miR)-135a protects against ISO-induced cardiac fibrosis by downregulating TRPM7 expression and currents. Astragaloside IV treatment also inhibited ISO-induced cardiac fibrosis by targeting the miR-135a-TRPM7-TGF- $\beta$ /Smad pathway $(84,85)$. These results provided a better understanding of the potential roles of TRPM7 in oxidative stress-induced cardiac fibrosis and suggested that TRPM7 channels can be regarded as a therapeutic target.

TRPM7 is involved in neurohormone activation during cardiac fibrosis. Ang II, a pro fibrogenic cell growth factor, plays a significant role in the occurrence and development of cardiac fibrosis (86). The relationship between TRPM7 and Ang II is complex. Ang II reduced TRPM7 kinase expression in the heart and aorta of mice (23), whereas it increased TRPM7 expression in rat ventricular CFs in a concentration-dependent manner (53). In TRPM7 kinase-deficient mice, Ang II-induced cardiac hypertrophy, interstitial fibrosis and left ventricular dysfunction are amplified (23). However, downregulation of TRPM7 attenuated Ang II-induced CF proliferation, differentiation, ECM production and accumulation $(53,87)$. The TRPM7 $\mathrm{Ca}^{2+}$ current, as well as the protein expression levels of TRPM7 and collagen III, initially increases and then later decreases under optimal Ang II concentrations for inducing cardiac fibrosis (88). Therefore, in different stages of Ang II-induced cardiac fibrosis, the role of TRPM7 may be different.

Similar effects in fibrotic changes in a rat sick sinus syndrome (SSS) model have been observed (89). The sinoatrial node and atria of SSS rats exhibit more fibers and higher expression levels of Ang II, TRPM7 and phosphorylated (p)-Smad2 and produce more collagen than sham rats (89). TRPM7 small interfering RNA (siRNA) inhibited Ang II-induced p-Smad2 expression and collagen synthesis in cardiac fibroblasts in vitro (89). The considerable inward flow of $\mathrm{Ca}^{2+}$ activated signaling pathways such as the TGF $\beta /$ Smad pathway, causing inflammation and cellular differentiation and thereby resulting in an acceleration of fibrosis (67). When exposure to Ang II is prolonged, the induction of cardiac fibroblast apoptosis and necrosis increases, possibly generating ulterior inflammatory reactions and fibrogenesis.

TGF- $\beta 1$ is a profibrotic factor which can stimulate the proliferation of fibroblasts. TGF- $\beta /$ Smad signaling is regarded to be the main pathway leading to tissue fibrosis in a number of diseases (90-92). TGF- $\beta 1$ can promote cardiac fibrosis by phosphorylating downstream Smad2/3, while activated Smad7 can ameliorate fibrosis by triggering TGF- $\beta$ receptor I and Smad protein degradation (93). Studies have found that the TRPM7 channel is also an important downstream target of TGF- $\beta 1$ induced liver (92), lung (94) and atrial fibrosis (67). TGF- $\beta 1$ induced differentiation of cultured human atrial fibroblasts is correlated with an increase of TRPM7 expression induced by TGF- $\beta 1$ (67). In addition, inhibition of TRPM7-mediated $\mathrm{Ca}^{2+}$ influx renders fibroblasts less sensitive to TGF- $\beta 1$ induced proliferation and differentiation (67), indicating that TRPM7-mediated $\mathrm{Ca}^{2+}$ signal is necessary for TGF- $\beta 1$ elicited fibrogenesis. Interestingly, latest research confirmed that TRPM7 gene silencing significantly suppressed the expression of TGF- $\beta 1$ and p-smad3, while the expression of Smad7 protein was increased (84). In addition, SB431542 (a TGF- $\beta 1$ blocker) significantly inhibited the expression of TRPM7 protein and currents in CFs (84). Thus, positive feedback occurs between enhanced TRPM7 function and TGF- $\beta /$ Smad pathway activation, which promotes cardiac fibrosis progression.

\section{Modulators for TRPM7}

Due to the wide range of physiological and pathophysiological roles ascribed to TRPM7, reliable drug-like molecules allowing distinction of the channel versus kinase activity in situ and under in vivo conditions are urgently needed.

Several potent inhibitors of the TRPM7 channel have been identified, including a group of non-specific channel blockers such as SKF-96365 and 2-APB, natural compounds and metabolites including waixenicin A, quinine and sphingosine and several synthetic drug-like compounds (95). In adult rat ventricular CFs, 2-APB inhibited Ang II-induced $\mathrm{Ca}^{2+}$ influx and the expression of myocardial connective tissue growth factor, $\alpha$-SMA and CF proliferation (53). Pharmacological targeting of the TRPM7 channel in conjunction with genetic silencing of the entire TRPM7 protein or the comparative analysis of effects induced by structurally unrelated TRPM7 modulators were shown to be instrumental in uncovering new cellular functions of TRPM7 and assessing the therapeutic potential of anti-TRPM7 drugs $(95,96)$.

The first positive gating modulator of the TRPM7 channel, naltriben, an antagonist of $\delta$-opioid receptors, reversibly activated the TRPM7 channel without prior depletion of intracellular $\mathrm{Mg}^{2+}$ and even under conditions of low $\mathrm{PIP}_{2}(96)$. Naltriben is the prototype of type 1 activators, allowing induction of the TRPM7 channel independently of $\left[\mathrm{Mg}^{2+}\right]_{\mathrm{i}}(95)$. Moreover, mibefradil is able to stimulate TRPM7-mediated $\mathrm{Ca}^{2+}$ entry as well as TRPM7 currents with an $\mathrm{EC}_{50}$ of $53 \mathrm{M}$ (97). Mibefradil is a type 2 agonist acting on the TRPM7 channel in a $\left[\mathrm{Mg}^{2+}\right]_{\mathrm{i}}$ dependent manner (97). Type 1 agonists (naltriben) will stimulate $\mathrm{Mg}^{2+}$ and $\mathrm{Ca}^{2+}$ influx irrespective of cytosolic $\mathrm{Mg}^{2+}$ levels, whereas type 2 agonists (mibefradil) will act preferentially on cells with reduced intracellular $\mathrm{Mg}^{2+}$ content. Hence, it is worth investigating whether putative endogenous TRPM7 ligands act in a similar manner (95).

A set of small organic modulators of TRPM7 have been identified (95-97). These new compounds allow for the activation or inhibition of TRPM7 currents or modulate 


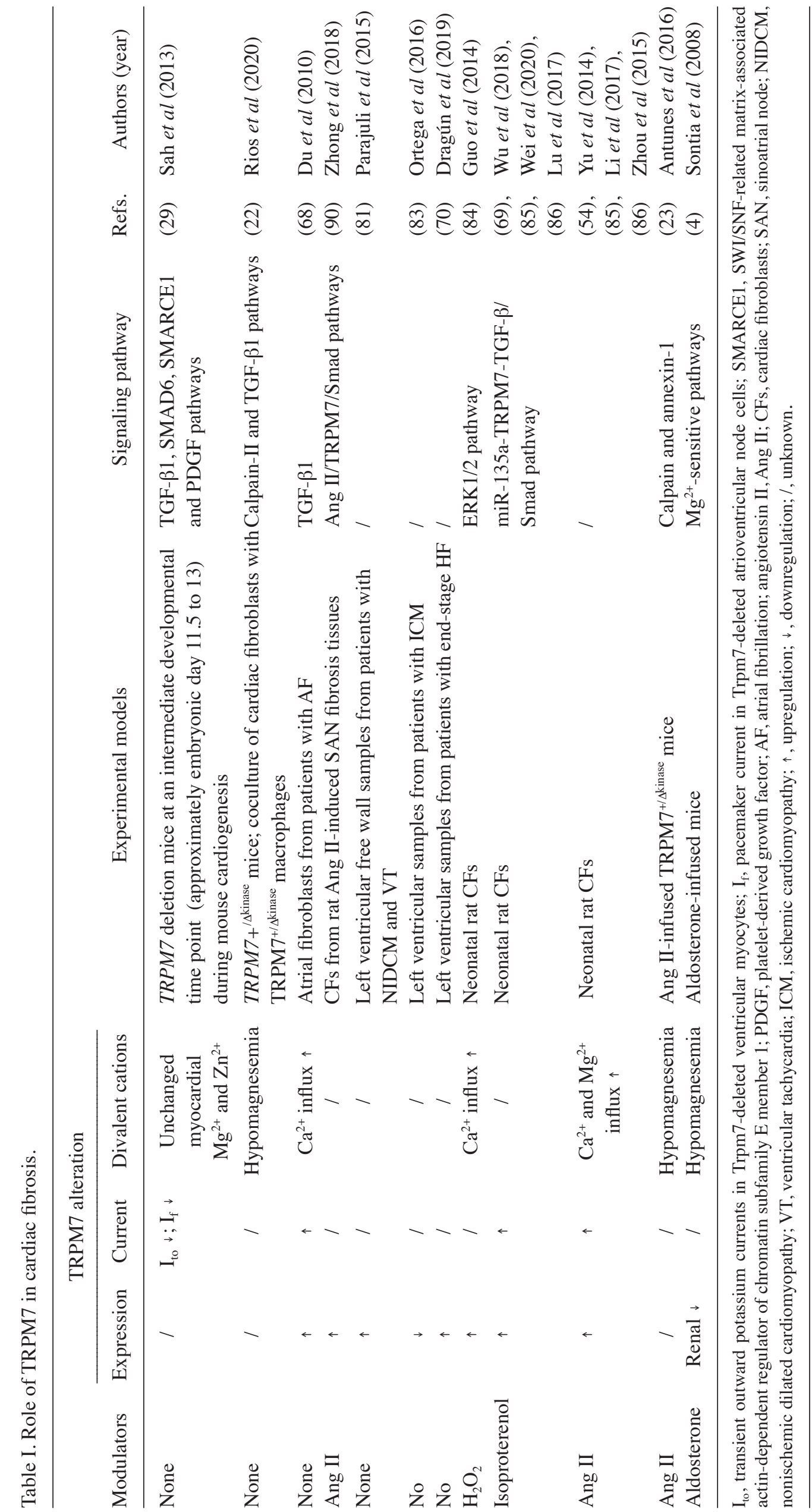


TRPM7 kinase activity (95-97). The newly identified TRPM7 modulators will undoubtedly be instrumental in deciphering the cellular functions of TRPM7. Small molecules that have been identified to date might be regarded as lead structures for further development of high-affinity in vivo drugs targeting TRPM7 (95).

\section{Implications and conclusion}

Myocardial fibrosis is a hallmark of cardiac remodeling and functionally contributes to the development of $\mathrm{HF}$, a leading cause of death worldwide (98). Clinically, no valid therapeutic agents target activated cardiac fibroblasts, owing to the extraordinary etiological heterogeneity and sophisticated pathogenesis of this disease. Blockade of TGF- $\beta 1$ signaling through pharmacological inhibition and magnetic nanoparticle targeted delivery of TGF- $\beta 1$ siRNA to ECs can relieve pathological cardiac fibrosis and hypertrophy in EC-forkhead box P1 deletion mice (6).

This growing evidence demonstrated that TRPM7 plays a nonredundant and vital regulatory role in activated cardiac fibroblasts through its ion channel function and/or kinase activity (Table I). In addition, the heart is a very compact organ comprising diverse cell types, and its pluricellularity offers the opportunity for intercellular communication within the heart. Fibroblasts create and sustain the biochemical and mechanical environment of the heart through their complex interactions with cardiomyocytes. Further investigations are needed to assess the interaction between TRPM7 in cardiac fibroblasts and cardiomyocytes or coronary microcirculatory ECs under different pathological disease models related to cardiac fibrogenesis.

In conclusion, these in vitro and in vivo findings suggested that TRPM7 is a potential pharmacological target for halting the development of fibrotic cardiac diseases. Future studies will provide more mechanistic insights into the role of TRPM7 in cardiac fibrosis.

\section{Acknowledgements}

Not applicable.

\section{Funding}

The project was supported by funding from the National Natural Science Foundation of China (grant nos. 82000062, 81960015, 81960074 and 81500233), Science and Technology Planning Project of Jiangxi Province (grant no. 20161ACG70012), Natural Science Foundation of Jiangxi Province (grant no. 20192BAB205033) and Jiangxi Province academic and technical leaders Training Plan-Young Talents Project.

\section{Availability of data and materials}

Not applicable.

\section{Authors' contributions}

FHu participated in the review design and drafted the manuscript. ML revised the manuscript critically for important intellectual content and illustrated the figures. FHa revised the manuscript critically for important intellectual content. QZ and YZ contributed to preparation, editing and review of the manuscript. WZ and XC participated in review design and contributed to quality control of data and images. $\mathrm{XC}$ gave final approval of the version to be published. All authors read and approved the final manuscript.

\section{Ethics approval and consent to participate}

Not applicable.

\section{Patient consent for publication}

Not applicable.

\section{Competing interests}

The authors declare that they have no competing interests.

\section{References}

1. Weber KT, Sun Y and Díez J: Fibrosis: A living tissue and the infarcted heart. J Am Coll Cardiol 52: 2029-2031, 2008.

2. Khan R and Sheppard R: Fibrosis in heart disease: Understanding the role of transforming growth factor-beta in cardiomyopathy, valvular disease and arrhythmia. Immunology 118: 10-24, 2006.

3. Klesen A, Jakob D, Emig R, Kohl P, Ravens U and Peyronnet R: Cardiac fibroblasts: Active players in (atrial) electrophysiology? Herzschrittmacherther Elektrophysiol 29: 62-69, 2018.

4. Sontia B, Montezano AC, Paravicini T, Tabet F and Touyz RM: Downregulation of renal TRPM7 and increased inflammation and fibrosis in aldosterone-infused mice: Effects of magnesium. Hypertension 51: 915-921, 2008.

5. Leask A: Getting to the heart of the matter: New insights into cardiac fibrosis. Circ Res 116: 1269-1276, 2015.

6. Liu J, Zhuang T, Pi J, Chen X, Zhang Q, Li Y, Wang H, Shen Y, Tomlinson B,ChanP,etal:EndothelialForkheadBox Transcription Factor P1 Regulates Pathological Cardiac Remodeling Through Transforming Growth Factor- $\beta 1$-Endothelin-1 Signal Pathway. Circulation 140: 665-680, 2019.

7. Frangogiannis NG: Fibroblasts and the extracellular matrix in right ventricular disease. Cardiovasc Res 113: 1453-1464, 2017.

8. Travers JG, Kamal FA, Robbins J, Yutzey KE and Blaxall BC: Cardiac Fibrosis: The Fibroblast Awakens. Circ Res 118: 1021-1040, 2016.

9. Moore-Morris T, Guimarães-Camboa N, Banerjee I, Zambon AC, Kisseleva T, Velayoudon A, Stallcup WB, Gu Y, Dalton ND, Cedenilla M, et al: Resident fibroblast lineages mediate pressure overload-induced cardiac fibrosis. J Clin Invest 124: 2921-2934, 2014.

10. Zeisberg EM, Tarnavski O, Zeisberg M, Dorfman AL, McMullen JR, Gustafsson E, Chandraker A, Yuan X, Pu WT, Roberts $\mathrm{AB}$, et al: Endothelial-to-mesenchymal transition contributes to cardiac fibrosis. Nat Med 13: 952-961, 2007.

11. Quijada P, Trembley MA and Small EM: The Role of the Epicardium During Heart Development and Repair. Circ Res 126: 377-394, 2020.

12. Simões FC, Cahill TJ, Kenyon A, Gavriouchkina D, Vieira JM, Sun X, Pezzolla D, Ravaud C, Masmanian E, Weinberger M, et al: Macrophages directly contribute collagen to scar formation during zebrafish heart regeneration and mouse heart repair. Nat Commun 11: 600, 2020.

13. Sundberg C, Ivarsson M, Gerdin B and Rubin K: Pericytes as collagen-producing cells in excessive dermal scarring. Lab Invest 74: 452-466, 1996.

14. Samanta A, Hughes TET and Moiseenkova-Bell VY: Transient Receptor Potential (TRP) Channels. Subcell Biochem 87: 141-165, 2018.

15. Kraft R and Harteneck C: The mammalian melastatin-related transient receptor potential cation channels: An overview. Pflugers Arch 451: 204-211, 2005. 
16. Clark K, Middelbeek J, Morrice NA, Figdor CG, Lasonder E and van Leeuwen FN: Massive autophosphorylation of the Ser/Thr-rich domain controls protein kinase activity of TRPM6 and TRPM7. PLoS One 3: e1876, 2008.

17. Li M, Jiang J and Yue L: Functional characterization of homoand heteromeric channel kinases TRPM6 and TRPM7. J Gen Physiol 127: 525-537, 2006.

18. Zhang Z, Yu H, Huang J, Faouzi M, Schmitz C, Penner R and Fleig A: The TRPM6 kinase domain determines the Mg.ATP sensitivity of TRPM7/M6 heteromeric ion channels. J Biol Chem 289: 5217-5227, 2014.

19. Hofmann T, Chubanov V, Gudermann T and Montell C: TRPM5 is a voltage-modulated and $\mathrm{Ca}\left({ }^{2+}\right)$-activated monovalent selective cation channel. Curr Biol 13: 1153-1158, 2003.

20. Fonfria E, Murdock PR, Cusdin FS, Benham CD, Kelsell RE and McNulty S: Tissue distribution profiles of the human TRPM cation channel family. J Recept Signal Transduct Res 26: 159-178, 2006.

21. Yue Z, Zhang Y, Xie J, Jiang J and Yue L: Transient receptor potential (TRP) channels and cardiac fibrosis. Curr Top Med Chem 13: 270-282, 2013.

22. Rios FJ, Zou ZG, Harvey AP, Harvey KY, Nosalski R, Anyfanti P, Camargo LL, Lacchini S, Ryazanov AG, Ryazanova L, et al: Chanzyme TRPM7 protects against cardiovascular inflammation and fibrosis. Cardiovasc Res 116: 721-735, 2020.

23. Antunes TT, Callera GE, He Y, Yogi A, Ryazanov AG Ryazanova LV, Zhai A, Stewart DJ, Shrier A and Touyz RM: Transient Receptor Potential Melastatin 7 Cation Channel Kinase: New Player in Angiotensin II-Induced Hypertension. Hypertension 67: 763-773, 2016.

24. Clapham DE: TRP channels as cellular sensors. Nature 426 : 517-524, 2003

25. Duan J, Li Z, Li J, Hulse RE, Santa-Cruz A, Valinsky WC, Abiria SA, Krapivinsky G, Zhang J and Clapham DE: Structure of the mammalian TRPM7, a magnesium channel required during embryonic development. Proc Natl Acad Sci USA 115 E8201-E8210, 2018.

26. Zou ZG, Rios FJ, Montezano AC and Touyz RM: TRPM7, Magnesium, and Signaling. Int J Mol Sci 20: 1877, 2019.

27. Suzuki S, Lis A, Schmitz C, Penner R and Fleig A: The TRPM7 kinase limits receptor-induced calcium release by regulating heterotrimeric G-proteins. Cell Mol Life Sci 75: 3069-3078, 2018.

28. Nadolni W and Zierler S: The Channel-Kinase TRPM7 as Nove Regulator of Immune System Homeostasis. Cells 7: 109, 2018.

29. Sah R, Mesirca P, Mason X, Gibson W, Bates-Withers C, Van den Boogert M, Chaudhuri D, Pu WT, Mangoni ME and Clapham DE: Timing of myocardial trpm7 deletion during cardiogenesis variably disrupts adult ventricular function, conduction, and repolarization. Circulation 128: 101-114, 2013.

30. Huang Y, Leng TD, Inoue K, Yang T, Liu M, Horgen FD, Fleig A, Li J and Xiong ZG: TRPM7 channels play a role in high glucose-induced endoplasmic reticulum stress and neuronal cell apoptosis. J Biol Chem 293: 14393-14406, 2018.

31. Chiang YF, Chen HY, Lee IT, Chien LS, Huang JH, Kolisek M, Cheng FC and Tsai SW: Magnesium-responsive genes are downregulated in diabetic patients after a three-month exercise program on a bicycle ergometer. J Chin Med Assoc 82: 495-499, 2019.

32. Sun HS: Role of TRPM7 in cerebral ischaemia and hypoxia. J Physiol 595: 3077-3083, 2017.

33. Chen M, Zhang W, Shi J and Jiang S: TGF- $\beta 1$-Induced Airway Smooth Muscle Cell Proliferation Involves TRPM7-Dependent Calcium Influx via TGFßR/SMAD3. Mol Immunol 103: 173-181, 2018.

34. Voringer S, Schreyer L, Nadolni W, Meier MA, Woerther K, Mittermeier C, Ferioli S, Singer S, Holzer K, Zierler S, et al: Inhibition of TRPM7 blocks MRTF/SRF-dependent transcriptional and tumorigenic activity. Oncogene 39: 2328-2344, 2020.

35. Yee NS: Role of TRPM7 in Cancer: Potential as Molecular Biomarker and Therapeutic Target. Pharmaceuticals (Basel) 10: 39, 2017.

36. Lal N, Bhardwaj S, Lalgudi Ganesan S, Sharma R and Jain P: Case of hypomagnesemia with secondary hypocalcemia with a novel TRPM6 mutation. Neurol India 66: 1795-1800, 2018.

37. Ryazanova LV, Rondon LJ,Zierler S, Hu Z, Galli J, Yamaguchi TP, Mazur A, Fleig A and Ryazanov AG: TRPM7 is essential for $\mathrm{Mg}\left({ }^{2+}\right)$ homeostasis in mammals. Nat Commun 1: 109, 2010.
38. Wang Y, Chen L, Wang K, Da Y, Zhou M, Yan H, Zheng D, Zhong S, Cai S, Zhu H, et al: Suppression of TRPM2 reduces renal fibrosis and inflammation through blocking TGF- $\beta 1$-regulated JNK activation. Biomed Pharmacother 120: 109556, 2019.

39. Nadler MJ, Hermosura MC, Inabe K, Perraud AL, Zhu Q, Stokes AJ, Kurosaki T, Kinet JP,Penner R, Scharenberg AM, et al: LTRPC7 is a Mg.ATP-regulated divalent cation channel required for cell viability. Nature 411: 590-595, 2001.

40. Chokshi R, Matsushita M and Kozak JA: Sensitivity of TRPM7 channels to $\mathrm{Mg}^{2+}$ characterized in cellfree patches of Jurkat T lymphocytes. Am J Physiol Cell Physiol 302: C1642-C1651, 2012.

41. Runnels LW, Yue L and Clapham DE: The TRPM7 channel is inactivated by PIP(2) hydrolysis. Nat Cell Biol 4: 329-336, 2002.

42. Deason-Towne F, Perraud AL and Schmitz C: Identification of Ser/Thr phosphorylation sites in the C2-domain of phospholipase C $\gamma 2$ (PLC $\gamma 2$ ) using TRPM7-kinase. Cell Signal 24: 2070-2075, 2012

43. Abiria SA, Krapivinsky G, Sah R, Santa-Cruz AG, Chaudhuri D, Zhang J, Adstamongkonkul P, DeCaen PG and Clapham DE: TRPM7 senses oxidative stress to release $\mathrm{Zn}^{2+}$ from unique intracellular vesicles. Proc Natl Acad Sci USA 114: E6079-E6088, 2017.

44. Clark K, Langeslag M, van Leeuwen B, Ran L, Ryazanov AG, Figdor CG, Moolenaar WH, Jalink K and van Leeuwen FN: TRPM7, a novel regulator of actomyosin contractility and cell adhesion. EMBO J 25: 290-301, 2006.

45. Clark K, Middelbeek J, Dorovkov MV, Figdor CG, Ryazanov AG Lasonder E and van Leeuwen FN: The alpha-kinases TRPM6 and TRPM7, but not eEF-2 kinase, phosphorylate the assembly domain of myosin IIA, IIB and IIC. FEBS Lett 582: 2993-2997, 2008.

46. Clark K, Middelbeek J, Lasonder E, Dulyaninova NG, Morrice NA, Ryazanov AG, Bresnick AR, Figdor CG and van Leeuwen FN: TRPM7 regulates myosin IIA filament stability and protein localization by heavy chain phosphorylation. J Mol Biol 378: 790-803, 2008.

47. Dorovkov MV and Ryazanov AG: Phosphorylation of annexin I by TRPM7 channel-kinase. J Biol Chem 279: 50643-50646, 2004.

48. Dorovkov MV, Kostyukova AS and Ryazanov AG: Phosphorylation of annexin A1 by TRPM7 kinase: A switch regulating the induction of an $\alpha$-helix. Biochemistry 50: 2187-2193, 2011.

49. Krapivinsky G,Krapivinsky L, Manasian Y and Clapham DE: The TRPM7 chanzyme is cleaved to release a chromatin-modifying kinase. Cell 157: 1061-1072, 2014.

50. Ryazanova LV, Dorovkov MV, Ansari A and Ryazanov AG: Characterization of the protein kinase activity of TRPM7/ChaK1, a protein kinase fused to the transient receptor potential ion channel. J Biol Chem 279: 3708-3716, 2004.

51. Matsushita M, Kozak JA, Shimizu Y, McLachlin DT, Yamaguchi H, Wei FY, Tomizawa K, Matsui H, Chait BT, Cahalan MD, et al: Channel function is dissociated from the intrinsic kinase activity and autophosphorylation of TRPM7/ChaK1. J Biol Chem 280: 20793-20803, 2005.

52. Desai BN, Krapivinsky G, Navarro B, Krapivinsky L, Carter BC, Febvay S, Delling M, Penumaka A, Ramsey IS, Manasian Y, et al: Cleavage of TRPM7 releases the kinase domain from the ion channel and regulates its participation in Fas-induced apoptosis. Dev Cell 22: 1149-1162, 2012

53. Yu Y, Chen S, Xiao C, Jia Y, Guo J, Jiang J and Liu P: TRPM7 is involved in angiotensin II induced cardiac fibrosis development by mediating calcium and magnesium influx. Cell Calcium 55 : 252-260, 2014.

54. Song C, Bae Y, Jun J, Lee H, Kim ND, Lee KB, Hur W, Park JY and Sim T: Identification of TG100-115 as a new and potent TRPM7 kinase inhibitor, which suppresses breast cancer cell migration and invasion. Biochim Biophys Acta Gen Subj 1861: 947-957, 2017.

55. Yang CW, Liu H, Li XD, Sui SG and Liu YF: Salvianolic acid B protects against acute lung injury by decreasing TRPM6 and TRPM7 expressions in a rat model of sepsis. J Cell Biochem 119: 701-711, 2018.

56. Liu A, Wu J, Yang C, Wu Y, Zhang Y, Zhao F, Wang H, Yuan L, Song L, Zhu T, et al: TRPM7 in CHBP-induced renoprotection upon ischemia reperfusion-related injury. Sci Rep 8: 5510, 2018.

57. Al Hattab D and Czubryt MP: A primer on current progress in cardiac fibrosis. Can J Physiol Pharmacol 95: 1091-1099, 2017.

58. Jalife J and Kaur K: Atrial remodeling, fibrosis, and atrial fibrillation. Trends Cardiovasc Med 25: 475-484, 2015. 
59. Schirone L, Forte M, Palmerio S, Yee D, Nocella C, Angelini F, Pagano F, Schiavon S, Bordin A, Carrizzo A, et al: A Review of the Molecular Mechanisms Underlying the Development and Progression of Cardiac Remodeling. Oxid Med Cell Longev 2017: 3920195, 2017.

60. Yue L, Xie J and Nattel S: Molecular determinants of cardiac fibroblast electrical function and therapeutic implications for atrial fibrillation. Cardiovasc Res 89: 744-753, 2011.

61. Zhang YH, Sun HY, Chen KH, Du XL, Liu B, Cheng LC, Li X, Jin MW and Li GR: Evidence for functional expression of TRPM7 channels in human atrial myocytes. Basic Res Cardiol 107: 282, 2012.

62. González A, López B and Díez J: Fibrosis in hypertensive heart disease: Role of the renin-angiotensin-aldosterone system. Med Clin North Am 88: 83-97, 2004

63. Olson ER, Shamhart PE, Naugle JE and Meszaros JG: Angiotensin II-induced extracellular signal-regulated kinase 1/2 activation is mediated by protein kinase Cdelta and intracellular calcium in adult rat cardiac fibroblasts. Hypertension 51: 704-711, 2008.

64. Manabe I, Shindo T and Nagai R: Gene expression in fibroblasts and fibrosis: Involvement in cardiac hypertrophy. Circ Res 91: 1103-1113, 2002.

65. Parekh AB: Calcium signalling in health and disease. Semin Cell Dev Biol 94: 1-2, 2019.

66. Fan D, Takawale A, Lee J and Kassiri Z: Cardiac fibroblasts, fibrosis and extracellular matrix remodeling in heart disease. Fibrogenesis Tissue Repair 5: 15, 2012.

67. Du J, Xie J,Zhang Z, Tsujikawa H, Fusco D, Silverman D, Liang B and Yue L: TRPM7-mediated $\mathrm{Ca}^{2+}$ signals confer fibrogenesis in human atrial fibrillation. Circ Res 106: 992-1003, 2010.

68. Wu Y, Liu Y, Pan Y, Lu C, Xu H, Wang X, Liu T, Feng K and Tang Y: MicroRNA-135a inhibits cardiac fibrosis induced by isoproterenol via TRPM7 channel. Biomed Pharmacother 104: 252-260, 2018.

69. Dragún M, Gažová A, Kyselovič J, Hulman M and Mát'uš M: TRP Channels Expression Profile in Human End-Stage Heart Failure. Medicina (Kaunas) 55: 380, 2019.

70. Morgan JP, Erny RE, Allen PD, Grossman W and Gwathmey JK Abnormal intracellular calcium handling, a major cause of systolic and diastolic dysfunction in ventricular myocardium from patients with heart failure. Circulation 81: 121-132, 1990.

71. Altura BM, Kostellow AB, Zhang A, Li W, Morrill GA, Gupta RK and Altura BT: Expression of the nuclear factor-kappaB and proto-oncogenes c-fos and c-jun are induced by low extracellular $\mathrm{Mg}^{2+}$ in aortic and cerebral vascular smooth muscle cells: Possible links to hypertension, atherogenesis, and stroke. Am J Hypertens 16: 701-707, 2003.

72. Touyz RM and Yao G: Up-regulation of vascular and renal mitogen-activated protein kinases in hypertensive rats is normalized by inhibitors of the $\mathrm{Na}^{+} / \mathrm{Mg}^{2+}$ exchanger. Clin Sci (Lond) 105: 235-242, 2003.

73. Baldoli E and Maier JA: Silencing TRPM7 mimics the effects of magnesium deficiency in human microvascular endothelial cells. Angiogenesis15: 47-57, 2012

74. Bates-Withers C, Sah RandClapham DE: TRPM7, the $\mathrm{Mg}\left({ }^{2+}\right.$ inhibited channel and kinase. Adv Exp Med Biol 704: 173-183, 2011.

75. Montezano AC, Zimmerman D, Yusuf H, Burger D, Chignalia AZ, Wadhera V, van Leeuwen FN and Touyz RM: Vascular smooth muscle cell differentiation to an osteogenic phenotype involves TRPM7 modulation by magnesium. Hypertension 56: 453-462, 2010.

76. Schiffrin EL and Touyz RM: Calcium, magnesium, and oxidative stress in hyperaldosteronism. Circulation 111: 830-831, 2005.

77. Miller BA and Zhang W: TRP channels as mediators of oxidative stress. Adv Exp Med Biol 704: 531-544, 2011.

78. Sun Y: Myocardial repair/remodelling following infarction: Roles of local factors. Cardiovasc Res 81: 482-490, 2009.

79. Swynghedauw B: Molecular mechanisms of myocardial remodeling. Physiol Rev 79: 215-262, 1999.

80. Parajuli N, Valtuille L, Basu R, Famulski KS, Halloran PF, Sergi $C$ and Oudit GY: Determinants of ventricular arrhythmias in human explanted hearts with dilated cardiomyopathy. Eur J Clin Invest 45: 1286-1296, 2015
81. Demir T, Yumrutas O, Cengiz B, Demiryurek S, Unverdi H, Kaplan DS, Bayraktar R, Ozkul N and Bagc1 C: Evaluation of TRPM (transient receptor potential melastatin) genes expressions in myocardial ischemia and reperfusion. Mol Biol Rep 41: 2845-2849, 2014

82. Ortega A, Roselló-Lletí E, Tarazón E, Gil-Cayuela C, Lago F, González-Juanatey JR, Martinez-Dolz L, Portolés M and Rivera M: TRPM7 is down-regulated in both left atria and left ventricle of ischaemic cardiomyopathy patients and highly related to changes in ventricular function. ESC Heart Fail 3. 220-224, 2016

83. Guo JL, Yu Y, Jia YY, Ma YZ, Zhang BY, Liu PQ, Chen SR and Jiang JM: Transient receptor potential melastatin 7 (TRPM7) contributes to $\mathrm{H}_{2} \mathrm{O}_{2}$-induced cardiac fibrosis via mediating $\mathrm{Ca}\left({ }^{2+}\right)$ influx and extracellular signal-regulated kinase 1/2 (ERK1/2) activation in cardiac fibroblasts. J Pharmacol Sci 125: 184-192, 2014.

84. WeiY,WuY,FengK,ZhaoY,TaoR,XuHandTang Y: AstragalosideIV inhibits cardiac fibrosis via miR-135a-TRPM7-TGF- $\beta$ /Smads pathway. J Ethnopharmacol 249: 112404, 2020.

85. Lu J, Wang QY, Zhou Y, Lu XC, Liu YH, Wu Y, Guo Q, Ma YT and Tang YQ: AstragalosideIV against cardiac fibrosis by inhibiting TRPM7 channel. Phytomedicine 30: 10-17, 2017.

86. Olson ER, Shamhart PE, Naugle JE and Meszaros JG: Angiotensin II-induced extracellular signal-regulated kinase $1 / 2$ activation is mediated by protein kinase Cdelta and intracellular calcium in adult rat cardiac fibroblasts. Hypertension 51: 704-711, 2008.

87. Li S, Li M, Yi X, Guo F, Zhou Y, Chen S and Wu X: TRPM7 channels mediate the functional changes in cardiac fibroblasts induced by angiotensin II. Int J Mol Med 39: 1291-1298, 2017.

88. Zhou Y, Yi X, Wang T and Li M: Effects of angiotensin II on transient receptor potential melastatin 7 channel function in cardiac fibroblasts. Exp Ther Med 9: 2008-2012, 2015.

89. Zhong H, Wang T, Lian G, Xu C, Wang H and Xie L: TRPM7 regulates angiotensin II-induced sinoatrial node fibrosis in sick sinus syndrome rats by mediating Smad signaling. Heart Vessels 33: 1094-1105, 2018

90. Xu F, Liu C, Zhou D and Zhang L: TGF- $\beta /$ SMAD Pathway and Its Regulation in Hepatic Fibrosis. J Histochem Cytochem 64 157-167, 2016.

91. Liu KH, Zhou N, Zou Y, Yang YY, OuYang SX and Liang YM: Spleen Tyrosine Kinase (SYK) in the Progression of Peritoneal Fibrosis Through Activation of the TGF- $\beta 1 /$ Smad3 Signaling Pathway. Med Sci Monit 25: 9346-9356, 2019.

92. Penke LR and Peters-Golden M: Molecular determinants of mesenchymal cell activation in fibroproliferative diseases. Cell Mol Life Sci 76: 4179-4201, 2019.

93. Overstreet JM, Samarakoon R, Meldrum KK and Higgins PJ: Redox control of $\mathrm{p} 53$ in the transcriptional regulation of TGF- $\beta 1$ target genes through SMAD cooperativity. Cell Signal 26: $1427-1436,2014$.

94. Yu M, Huang C, Huang Y, Wu X, Li X and Li J: Inhibition of TRPM7 channels prevents proliferation and differentiation of human lung fibroblasts. Inflamm Res 62: 961-970, 2013

95. Chubanov V, Ferioli S and Gudermann T: Assessment of TRPM7 functions by drug-like small molecules. Cell Calcium 67: 166-173, 2017.

96. Hofmann T, Schäfer S, Linseisen M, Sytik L, Gudermann T and Chubanov V: Activation of TRPM7 channels by small molecules under physiological conditions. Pflugers Arch 466: 2177-2189, 2014.

97. Schäfer S, Ferioli S, Hofmann T, Zierler S, Gudermann T and Chubanov V: Mibefradil represents a new class of benzimidazole TRPM7 channel agonists. Pflugers Arch 468: 623-634, 2016.

98. Tanai E and Frantz S: Pathophysiology of Heart Failure. Compr Physiol 6: 187-214, 2015.

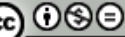

This work is licensed under a Creative Commons Attribution-NonCommercial-NoDerivatives 4.0 International (CC BY-NC-ND 4.0) License. 